\title{
Aprender a enseñar matemáticas en la formación inicial de profesores de educación especial
}

\section{Learning to teach mathematics in special education teacher initial training}

Aprendendo a ensinar matemática na formação inicial de professores de educação especial

\author{
Juan Luis Piñeiro \\ Universidad Metropolitana de Ciencias de la Educación \\ Santiago, Chile \\ E-mail: juanluis.pineiro@umce.cl \\ Orcid: 0000-0002-9616-3925
}

Resumen: En este trabajo se presenta el diseño de una asignatura para el plan formativo de profesores de educación especial. Comenzamos describiendo brevemente los fundamentos teóricos qué se centran prácticas de enseñanza efectivas y el Análisis Didáctico desde la perspectiva del Grupo de Investigación FQM-193. Posteriormente, explicitamos la estructura de la actividad curricular, describiendo y ejemplificando las actividades realizadas. Finalmente, reseñamos las conjeturas que subyacen en la propuesta.

Palabras clave: Formación inicial de profesores. Educación especial. Educación matemática. 
Abstract: In this paper, we present the design of a subject for the training plan of special education teachers. We begin by briefly describing the theoretical foundations that focus on effective teaching practices and Didactic Analysis from the perspective of the FQM.93 Research Group. Subsequently, we explain the structure of the subject, describing and exemplifying the activities carried out. Finally, we outline the conjectures underlying the proposal.

Keywords: Teacher training. Special Education. Mathematics education.

Resumo: Neste artigo apresentamos a concepção de um tema para o plano de treinamento para professores de educação especial. Começamos descrevendo brevemente os fundamentos teóricos que se concentram em práticas de ensino eficazes e Análise Didática a partir da perspectiva do Grupo de Pesquisa FQM.93. Em seguida, explicamos a estrutura da atividade curricular, descrevendo e exemplificando as atividades realizadas. Finalmente, delineamos as conjecturas subjacentes à proposta.

Palavras-chave: Treinamento inicial de professores. Educação especial. Educação em matemática.

Recebido em $19 / 07 / 2021$

Aceito em $18 / 10 / 2021$ 


\section{Universidade Federal da Grande Dourados}

\section{INTRODUCCIÓN}

La preparación de los profesores de Educación Especial (EE) para enseñar matemáticas ha sido una de las áreas que menor atención ha tenido en algunos programas de formación inicial, con un número menor de oportunidades para aprender matemáticas (Greer y Meyen, 2009). La literatura señala que cuándo los académicos de EE discuten lo que los futuros profesores necesitan saber, tienden a enfocarse en el conocimiento de las prácticas basadas en evidencia, experimentos de enseñanza, el monitoreo del progreso para tomar decisiones en la enseñanza y la colaboración efectiva con otros profesionales y padres (Park, Kiely, Brownell, y Benedict, 2019). Por ejemplo, López-Mojica (2016) señala que la formación matemática de los profesores de EE en México no se extiende más de un curso o una unidad dentro de un semestre y está centrada en la construcción del número natural y sus operaciones.

Lo anterior sugiere que el papel que desempeña del conocimiento disciplinar en la enseñanza en la EE a menudo no se discute, y solo se ha investigado en un número menor de estudios centrados mayormente en la lectura, en desmedro del conocimiento sobre las matemáticas (Griffin, van Garderen y Ulrich, 2014). No obstante, el conocimiento matemático para la enseñanza que el docente posea, ha sido señalado como uno de los elementos indicativos de la calidad del aprendizaje de los estudiantes, ya que las actividades que realice en el aula dependen en gran medida de este (Brownell et al., 2014). En definitiva, el conocimiento de las matemáticas es un tema relegado y que la literatura muestra como problemático para los profesores de EE y especialmente en la formación inicial.

En este contexto, este trabajo presenta el diseño de una experiencia para aprender a enseñar matemáticas en la formación inicial de profesores de EE en una universidad pública chilena. Se comienza presentando el contexto y antecedentes que justifican y hacen emerger la propuesta. Luego se continúa presentando sus bases teóricas. Posteriormente, se describen las actividades realizadas, para terminar con consideraciones respecto al diseño. 


\section{Contexto y ANTECEDENTES}

El año 2016, la Universidad Metropolitana de Ciencias de la Educación (UMCE) oficializó su nuevo modelo educativo (Vicerrectoría Académica, 2016). En este documento se presenta un "enfoque global de significados compartidos por la comunidad sobre diversos componentes propios de la formación" (Op. Cite., p. 7). Esto se tradujo en seis principios orientadores, a saber, Transformacional, Integralidad, Flexibilidad, Reflexibilidad, Proyectividad y Plasticidad.

A raíz de la actualización del modelo educativo, la UMCE comenzó un proceso de rediseño curricular a todas su formación, tanto de pregrado como de postgrado. Particularmente, la carrera EE especialidad en Comunicación y Lenguaje, o en Educación de Personas Sordas, o en Aprendizaje ha rediseñado su plan de estudios en torno a cuatro áreas de saber: desarrollo humano y diversidad, comunicación y aprendizaje, didáctica y evaluación, y desarrollo profesional docente ( $U G C l, 2019$ ). Concretamente, la actividad curricular que aquí se describe adscribe a las áreas Didáctica y Evaluación y se articula con una Práctica que lleva a los futuros profesores a aulas de educación primaria.

La actividad curricular se ubica en el V semestre de la carrera y posee 4 SCT (Sistema de Créditos Transferibles Chile), que se traduce en 3 horas presenciales y 2 horas de trabajo autónomo por 18 semanas. Fue realizada en dos secciones: una sección con las especialidades de Personas Sordas y Lenguaje y Comunicación de 22 estudiantes; y otra sección con la especialidad Aprendizaje con 33 estudiantes. El resultado formativo esperado tiene relación con la planificación y diversificación de la enseñanza de las matemáticas.

\section{Perspectiva teórica}

La propuesta curricular que se describe aquí tiene como sustento teórico las prácticas efectivas en la enseñanza de las matemáticas (NCTM, 2014), las prácticas que Montague y Jitendra (2012) identificaron con mayor impacto para la enseñanza 
de estudiantes con dificultades con las matemáticas y el Análisis Didáctico (AD) desde la perspectiva del grupo de investigación "FQM-193. Didáctica de la Matemática. Pensamiento Numérico" del Departamento de Didáctica de la Matemática de la Universidad de Granada (Rico, Lupiáñez y Molina, 2013).

Las practicas efectivas en la enseñanza de las matemáticas son "ocho prácticas de enseñanza de las matemáticas proporcionan un marco teórico para fortalecer la enseñanza y el aprendizaje de las matemáticas" (NCTM, 2014, p. 9). La tabla 1 muestra las prácticas y sus descripciones. Estas ideas son planteadas teniendo en cuenta la literatura tanto cognitiva como de educación matemática.

\section{Tabla 1}

Práctica de enseñanza de las matemáticas.

\begin{tabular}{ll}
\hline \multicolumn{1}{c}{ Práctica } & \multicolumn{1}{c}{ Descripción } \\
\hline $\begin{array}{l}\text { Establecimiento de } \\
\text { metas matemáticas } \\
\text { enfocadas en el } \\
\text { aprendizaje }\end{array}$ & $\begin{array}{l}\text { Una enseñanza eficaz de las matemáticas establece metas } \\
\text { matemáticas claras concernientes con las matemáticas que los } \\
\text { estudiantes están aprendiendo, las inserta dentro de los desarrollos de } \\
\text { aprendizaje y las utiliza como guía para las decisiones de enseñanza. }\end{array}$ \\
\hline $\begin{array}{l}\text { Implementación de } \\
\text { tareas que promuevan } \\
\text { el razonamiento y la } \\
\text { resolución de } \\
\text { problemas }\end{array}$ & $\begin{array}{l}\text { La enseñanza eficaz de las matemáticas involucra a los estudiantes en } \\
\text { tareas de resolución y análisis, las cuales promueven el razonamiento } \\
\text { matemático y la resolución de problemas, además de que permiten que } \\
\text { haya múltiples maneras de abordar los problemas y existan estrategias } \\
\text { de resolución variadas. }\end{array}$ \\
\hline $\begin{array}{l}\text { Uso y vinculación de } \\
\text { las representaciones } \\
\text { matemáticas }\end{array}$ & $\begin{array}{l}\text { Una enseñanza eficaz de las matemáticas obliga a los estudiantes a } \\
\text { establecer conexiones entre diferentes representaciones matemáticas } \\
\text { para profundizar el entendimiento de conceptos y procedimientos } \\
\text { matemáticos, así como para concebir a ambos como herramientas para } \\
\text { la resolución de problemas. }\end{array}$ \\
$\begin{array}{l}\text { Favorecimiento del } \\
\text { discurso matemático } \\
\text { significativo }\end{array}$ & $\begin{array}{l}\text { Una enseñanza eficaz de las matemáticas promueve el diálogo entre } \\
\text { los estudiantes a fin de que puedan construir una comprensión } \\
\text { compartida de las ideas matemáticas, a través del análisis y la } \\
\text { comparación de sus enfoques y argumentos. }\end{array}$ \\
\hline $\begin{array}{l}\text { Planteamiento de } \\
\text { preguntas deliberadas }\end{array}$ & $\begin{array}{l}\text { Una enseñanza eficaz de las matemáticas utiliza preguntas deliberadas } \\
\text { para evaluar y mejorar el razonamiento del estudiante y para que le dé } \\
\text { sentido a ideas y relaciones matemáticas importantes. }\end{array}$ \\
\hline
\end{tabular}


Universidade Federal da Grande Dourados

\begin{tabular}{ll}
\hline \multicolumn{1}{c}{ Práctica } & \multicolumn{1}{c}{ Descripción } \\
\hline $\begin{array}{l}\text { Elaboración de la } \\
\text { fluidez procedimental a } \\
\text { partir de la } \\
\text { comprensión } \\
\text { conceptual }\end{array}$ & $\begin{array}{l}\text { Una enseñanza eficaz de las matemáticas logra la fluidez en los } \\
\text { procedimientos matemáticos basándose en la comprensión conceptual, } \\
\text { de manera que los estudiantes, con el tiempo, se vuelvan hábiles en el } \\
\text { empleo flexible de procedimientos, a medida que resuelven problemas } \\
\text { contextuales y matemáticos. }\end{array}$ \\
\hline $\begin{array}{l}\text { Favorecer el esfuerzo } \\
\text { productivo en el } \\
\text { aprendizaje de las } \\
\text { matemáticas }\end{array}$ & $\begin{array}{l}\text { Una enseñanza eficaz de las matemáticas brinda consistentemente a } \\
\text { los estudiantes, de manera individual y colectiva, las oportunidades y } \\
\text { los apoyos necesarios para que se involucren en esfuerzos productivos } \\
\text { a medida que aborden ideas y relaciones matemáticas. }\end{array}$ \\
\hline $\begin{array}{l}\text { Obtener y utilizar } \\
\text { evidencias del } \\
\text { pensamiento de los } \\
\text { estudiantes }\end{array}$ & $\begin{array}{l}\text { Una enseñanza eficaz de las matemáticas utiliza evidencia del } \\
\text { pensamiento del estudiante para evaluar el progreso en la comprensión } \\
\text { matemática y para adecuar continuamente la enseñanza en formas que } \\
\text { apoye y extienda el aprendizaje. }\end{array}$ \\
\hline
\end{tabular}

Fuente: NCTM, 2014, p. 10.

La segunda idea teórica adoptada es la de Montague y Jitendra (2012), que mediante un meta-análisis, identificaron 5 prácticas con mayor impacto para la enseñanza de estudiantes con dificultades con las matemáticas. A estas prácticas, en la actividad curricular se les ha denominado prácticas basadas en evidencia para la diversificación de la enseñanza. En orden de significatividad de sus efectos, estás son: enseñanza explicita; representaciones visuales; secuencia de ejemplos; verbalizaciones de los estudiantes; y retroalimentación continua. La tabla 2 muestra sus descripciones.

\section{Tabla 1}

Estrategias de enseñanza con mayor impacto para la enseñanza de estudiantes con dificultades de aprendizaje en matemáticas.

\begin{tabular}{ll}
\hline \multicolumn{1}{c}{ Práctica } & \multicolumn{1}{c}{ Descripción } \\
\hline $\begin{array}{l}\text { Enseñanza } \\
\text { explícita }\end{array}$ & $\begin{array}{l}\text { La enseñanza explícita trata de que (a) el profesor demuestra un plan } \\
\text { (estrategia) paso a paso para resolver un problema; (b) el plan es específico } \\
\text { para el problema y no una guía heurística genérica para resolver problemas; } \\
\text { y (c) se alienta activamente a los estudiantes a usar el mismo procedimiento } \\
\text { / pasos demostrados por el maestro. }\end{array}$ \\
\hline
\end{tabular}


Práctica

Representaciones visuales

\section{Descripción}

La enseñanza de representaciones visuales trata de la enseñanza específica y explícita de imágenes visuales para ser usadas como estrategias en la resolución de problemas. Al ser trabajada de forma aislada, esta estrategia obtiene buenos resultados. Sin embargo, se obtuvieron mejores efectos cuando se usaron en combinación con otras.

Secuencia de ejemplos

La enseñanza las matemáticas mediante la selección cuidadosa y la secuencia de ejemplos se basa principalmente en dos características: las tareas deben seguir una secuencia / patrón específico y deben representar una variedad sistemática en el rango de ejemplos.

Verbalizaciones de los estudiantes

Esta práctica de enseñanza trata del proceso de alentar a los estudiantes a verbalizar su pensamiento o sus estrategias, o incluso las estrategias explícitas modeladas por el profesor. El proceso de verbalizar cómo resolver problemas debe alentar a los estudiantes a seleccionar una representación adecuada, discutiendo con sus compañeros y/o el profesor para evaluar la relevancia de esta. Otro tipo de discusiones que pueden utilizarse son sobre qué estrategias se aplican a situaciones particulares.

Retroalimentación continua

La retroalimentación continua trata de proporcionar información específica sobre su desempeño a cada estudiante, así como a sus profesores.

Fuente: Montague y Jitendra (2012).

El AD desde la perspectiva del grupo de investigación "FQM193. Didáctica de la Matemática. Pensamiento Numérico" del Departamento de Didáctica de la Matemática de la Universidad de Granada (Rico et al., 2013). Esta perspectiva, el AD, ha ampliado sus funciones más allá de la planificación y diseño de unidades didácticas (Rico y Fernández-Cano, 2013). Concretamente, se observan otros tres ámbitos de actuación (Castro-Rodríguez, 2015). Primero, en el ámbito curricular, como nivel de reflexión sobre la estructura del currículo de matemáticas, necesario para su estudio y el trabajo sobre el mismo. Segundo, en el ámbito profesional, como estrategia de formación de profesorado. Tercero, en el ámbito investigativo, el $A D$ puede usarse como metodología de investigación de orientación cuantitativa o cualitativa en una primera instancia, y como meta-evaluación en una segunda instancia.

Dentro de cada una de estas funciones, el $A D$ es entendido como un procedimiento cíclico compuesto por cinco etapas, a cada una de las cuales 


\section{Universidade Federal da Grande Dourados}

corresponde un tipo de análisis particular: análisis conceptual, análisis del contenido matemático escolar, análisis cognitivo, análisis de instrucción y análisis de actuación o evaluativo. Cada una de ellas consiste en un proceso de análisis y síntesis, que identifica datos relevantes, a partir de los cuales cierra un ciclo y da paso a la siguiente fase como puede observarse en la figura 1.

Figura 1. Análisis didáctico.

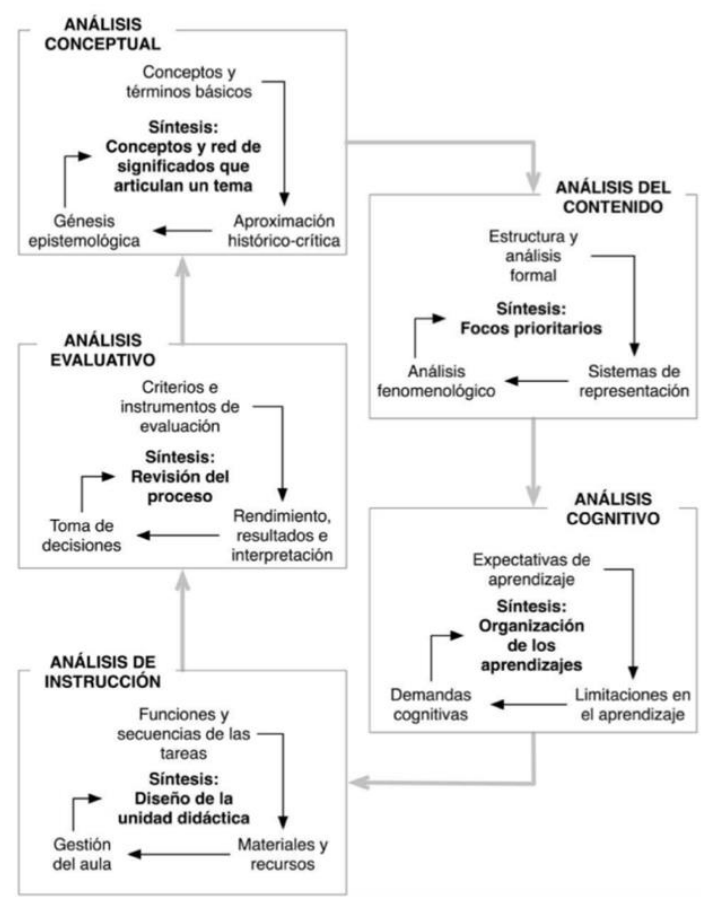

Fuente: Rico (2013, p. 22).

\section{LA ACTIVIDAD CURRICULAR}

El diseño que se presenta consta de dos núcleos (temas) de aprendizaje —una relativa a las prácticas efectivas generales, seguido de otro, sobre prácticas para la diversificación. En dichos núcleos se utilizó el AD para que en grupos elaboren, de manera colaborativa, una planificación que incluyan ambas perspectivas de prácticas de enseñanza. Así, se les solicitan a los futuros profesores realizar un AD a un contenido matemático escolar, finalizando con una planificación diversificada. Para el 
$A D$, se ha considerado que se focalice en un contenido matemático que se haya detectado más descendido en la prueba diagnóstica que se aplicó al iniciar la actividad curricular.

De esta manera se pretende que la formación relativa al conocimiento matemático para la enseñanza de futuras/os educadores especiales abarque un espectro mayor a solo la aritmética básica.

\section{Evaluación Diagnóstica}

La primera actividad del curso trató de una evaluación diagnóstica que indaga en los conocimientos matemáticos de los futuros docentes. Para aproximarse a esta tarea se ha utilizado el instrumento desarrollado por Martínez y colaboradores (2019). Este está compuesto de dos apartados: creencias respecto de la matemática, su enseñanza y aprendizaje; y Conocimiento Matemático Escolar (CME).

El primer apartado se compone de 47 ítems de escala Likert, donde para cada afirmación se solicita evaluar el grado de acuerdo asignando un valor entre 1 (muy en desacuerdo) y 4 (muy de acuerdo). Estos ítems se distribuyen en 15 relativos a la enseñanza y el aprendizaje; 19 sobre expectativas y logros; y 13 sobre la matemática.

El segundo apartado, relativo al CME evalúa los contenidos y las habilidades emanadas del currículo con el que los estudiantes para profesor comienzan su formación inicial. Para el desarrollo de este apartado, los autores se han basado en el currículo de educación primaria chileno (Ministerio de Educación, 2012) y en los dominios de habilidad de prueba TIMSS (Trends in International Mathematics and Science Study) (Mullis y Martin, 2013). Así, este apartado es estructurado de la siguiente manera: 20 corresponden a números y operaciones (11 a conocer, 8 a aplicar y 1 a razonar); 4 a medición (2 a conocer, 1 a aplicar y 1 a razonar); 7 a geometría ( 3 a conocer, 4 a aplicar y 0 a razonar); 4 a álgebra ( 0 a conocer, 2 a aplicar y 2 a razonar); y 5 a datos y probabilidades ( 0 a conocer, 4 a aplicar y 1 a razonar). 
Una vez aplicado el instrumento, cada estudiante recibió un informe con los resultados referidos al CME.

\section{ACTIVIDADES}

Análisis situaciones de aula. Una de las tareas que se solicitó a los futuros docentes tiene relación analizar diversas situaciones de aula que ilustraran cada una de las 8 prácticas de enseñanza (NCTM, 2014). Estas tareas podrían tomar dos perspectivas. La primera de ellas tiene relación con solicitar reflexionar sobre lo realizado por docentes, sus motivaciones y qué razones podrían fundamentar su acciones. La segunda se corresponde con dar sugerencias de cambio al docente de una situación analizada para incorporar la práctica de enseñanza que se estuviera trabajando en dicha clase. La tabla 3 muestra ejemplos de estas dos perspectivas.

\section{Tabla 3}

Ejemplos de preguntas para la reflexión.

\section{Práctica}

Uso y vinculación de las representaciones matemáticas
Ejemplos de preguntas

¿Qué contenido matemático se trabaja en la clase? ¿Qué tipos de representaciones aparecen en el relato? ¿Qué meta matemática puede inferirse del relato de clase? ¿A qué nivel cognitivo (Stein y Smith, 1998) se ajusta la tarea matemática propuesta por el profeso? Identifica (destacando o subrayando en el pdf) los momentos en que el profesor utiliza las estrategias sugeridas para desarrollar la competencia de sus estudiantes para representar.

Favorecimiento del discurso matemático significativo
¿Cómo contribuyó el profesor a la construcción de ideas matemáticas de los estudiantes? ¿Qué podría haber hecho el profesor, previo a la clase, para contribuir a la discusión de las ideas matemáticas?

Nombra tres acciones que harías para conducir una discusión significativa de las ideas matemáticas.

Análisis Didáctico. Está actividad es la tarea central de la actividad curricular y trataba de realizar un análisis de contenido, análisis cognitivo, análisis de instrucción y análisis evaluativo sobre un contenido matemático escolar. Para la selección del contenido se 
solicitó a los estudiantes utilizar los resultados de la prueba CME para formar los grupos que desarrollarían el AD. Esta solicitud se tradujo en que la sección 1 formalizó 10 grupos y la sección 2, 6 grupos. Las temáticas incluyeron: números naturales, aritmética de los números naturales, razonamiento lógico-matemático, patrones y álgebra, fracciones, números decimales, razones y proporciones, números enteros, geometría del plano y el espacio, estimación y medida, estadística y probabilidad.

Este trabajo fue realizado a modo de taller en uno de los dos bloques con que consta semanalmente la actividad curricular. La dinámica se realizó siguiendo una estructura de tutoría en la que el profesor de la actividad curricular retroalimentaba de manera particular a cada grupo sus dudas y consultas.

En una primera etapa se solicitó a los futuros profesores que realizarán un análisis del tratamiento curricular que recibía el contenido seleccionado en educación infantil y educación básica. Luego, se les pidió que seleccionaran y organizaran los significados del concepto matemático seleccionado y sus procedimientos mediante un análisis de contenido. Este significado del concepto matemático fue abordado a través de los tres componentes que articulan este análisis: estructura conceptual, sistemas de representación y contextos y modos de uso. En una tercera etapa realizaron un análisis cognitivo que giraba en torno a lo que el profesor espera que aprendan los escolares, lo que puede interferir en ese aprendizaje y lo que permite a los escolares aprender y al profesor observar si se produce ese aprendizaje de manera efectiva (Lupiáñez, 2013). Seguidamente, se solicitó realizar un análisis de instrucción en el que diseñaron, seleccionaron y secuenciaron las tareas que conformarían su planificación; asimismo, se les pidió identificar aspectos relativos a la gestión del aula y al empleo de materiales y recursos (Lupiáñez, 2013). Finalmente, realizaron un análisis de evaluación en el qué se debía valorar en qué medida se lograba lo que se pretendía y a qué aspectos se atribuyen los logros y las falencias de los diseños didácticos realizados (Lupiáñez, 2013). No obstante, debido a qué no se llevó al aula, se limitó establecer los criterios de evaluación y qué tareas matemáticas pueden utilizarse para evidenciar la comprensión por parte de los estudiantes. 


\section{Universidade Federal da Grande Dourados}

Planificación diversificada. La última actividad solicitada se trató de incorporar a los diseños didácticos, alguna de las prácticas identificadas cómo las que mayor impacto producen en los aprendizajes de los estudiantes con dificultades en el aprendizaje de las matemáticas. En esta tarea, además de presentar una planificación, los futuros profesores debían fundamentar las decisiones didácticas en torno a la utilización de las prácticas de enseñanza de las matemáticas (NCTM, 2014) y las prácticas basadas en evidencia (Montague y Jitendra, 2012).

\section{Consideraciones Finales}

La formación inicial de profesores de EE en el área de las matemáticas ha sufrido un fenómeno particular. Los estándares orientadores (que están en proceso de actualización) que fijan los conocimientos disciplinarios y pedagógicos de los futuros profesores de EE en Chile (Ministerio de Educación, 2014) son sumamente generales cuando se trata de conocimientos disciplinares. Hecho bastante similar a lo que sucede con la formación de estos profesores en otros países (Griffin et al., 2014). Concretamente, el documento chileno utiliza los estándares pedagógicos de educación primaria y de educación secundaria para orientar la formación de contenidos curriculares. Particularmente, en los estándares de conocimientos relativos a la educación primaria, se establece en el estándar 3 que el futuro profesor “conoce el currículum de Educación Básica (primaria) y usa sus diversos instrumentos curriculares para analizar y formular propuestas pedagógicas y evaluativas" (op. Cite., p. 17). Posteriormente, en el mismo documento se señala que este estándar se manifiesta cuándo el futuro profesor "identifica en el currículum de las cuatro asignaturas, los conceptos fundamentales y habilidades que necesita dominar para poder propiciar en sus futuros estudiantes los aprendizajes esperados" (op. Cite., p. 47). En este contexto cabe preguntarse, ¿qué conceptos y habilidades deberían enseñarse en la formación de profesores de EE relativos al currículo de las matemáticas en educación primaria? En caso de una respuesta afirmativa, ¿debe un profesor de EE tener la misma formación disciplinar y didáctica en el área de las 
matemáticas que un profesor de educación primaria? Más aún, en caso de ser positiva esta última, ¿qué formación deberían afrontar respecto al conocimiento disciplinar y didáctico de educación secundaria?

Si bien ninguna de estas preguntas tienen una respuesta directa y simple, el diseño aquí presentado tiene por objeto, por una parte, poner atención en la formación de los futuros profesores de EE sobre las matemáticas. Por otra, ampliar la perspectiva respecto a las oportunidades de aprender matemáticas de este colectivo. Este hecho sustentado en que el conocimiento de las matemáticas de los profesores es clave en el aprendizaje de los estudiantes (Hill, Rowan y Ball, 2005) e incluso en la calidad de las clases que puedan ofrecer los profesores (Hill et al., 2008). Esto sugiere que el conocimiento que sostengan los profesores de EE sobre las matemáticas tendrá un impacto en los estudiantes que atienden en las escuelas y colegios. No obstante, la problemática sobre qué matemáticas deberían conocer los profesores de EE es una línea abierta (Griffin et al., 2014).

\section{REFERENCIAS}

Brownell, M. T., Steinbrecher, T., Kimerling, J., Park, Y., Bae, J. y Benedict, A. (2014). Dimensions of teacher quality in general and special education. En P. T. Sindelar, E. D. McCray, M. T. Brownell y B. Lingnugaris/Kraft (Eds.), Handbook of research on special education teacher preparation (pp. 423444).

Castro-Rodríguez, E. (2015). Significados de las fracciones en las matemáticas escolares y formación inicial de maestros (Tesis doctoral). Universidad de Granada, España. 
Greer, D. L. y Meyen, E. L. (2009). Special education teacher education: A perspective on content knowledge. Learning Disabilities Research \& Practice, 24(4), 196-203.

Griffin, C. C., van Garderen, D. y Ulrich, T. G. (2014). Teacher preparation. Mathematics. En P. T. Sindelar, E. D. McCray, M. T. Brownell y B. Lignugaris/Kraft (Eds.), Handbook of research on special education teacher preparation (pp. 271-287). Nueva York, NY: Routledge.

Hill, H. C., Blunk, M. L., Charalambous, C. Y., Lewis, J. M., Phelps, G. C., Sleep, L. y Ball, D. L. (2008). Mathematical knowledge for teaching and the mathematical quality of instruction: An exploratory study. Cognition and Instruction, 26(4), 430-511.

Hill, H. C., Rowan, B. y Ball, D. L. (2005). Effects of teachers' mathematical knowledge for teaching on student achievement. American Educational Research Journal, 42(2), 371-406.

López-Mojica, J. M. (2016). Formación matemática de los docentes de educación especial. En J. Cuevas y A. I. Andrade (Eds.), Abordajes metodológicos para problemas educativos emergente (pp. 173-184). San Luis de Potosí, México: Centro de Estudios Jurídicos y Sociales Mispat y Universidad Autónoma de San Luis de Potosí.

Lupiáñez, J. L. (2013). Análisis didáctico. La planificación del aprendizaje desde una perspectiva curricular. En L. Rico, J. L. Lupiáñez y M. Molina (Eds.), Análisis didáctico en educación matemática (pp. 81-102). Madrid, España: Comares. 
Martínez, M. V., Rojas, F., Ulloa, R., Chandía, E., Ortiz, A. y Perdomo-Díaz, J. (2019). Creencias y conocimiento matemático escolar al comienzo de la formación inicial docente en estudiantes de Pedagogía General Básica. Pensamiento Educativo, 56(2), 1-19.

Ministerio de Educación. (2012). Bases curriculares Educación Básica. Santiago, Chile: Unidad de Curriculum y Evaluación.

Ministerio de Educación. (2014). Estándares orientadores para carreras de Educación Especial. Santiago, Chile: Autor.

Montague, M. y Jitendra, A. K. (2012). Research-based mathematics instruction for students with learning disabilities. En H. Forgasz y R. Rivera (Eds.), Towards equity in mathematics education: Gender, culture, and diversity (pp. 481-502). Cham, Suiza: Springer.

Mullis, I. V. S. y Martin, M. O. (2013). TIMSS 2015 assessment frameworks. Chestnut Hill, MA: IEA.

NCTM. (2014). Principles to actions: Ensuring mathematical success for all. Reston, VA: Autor.

Park, Y., Kiely, M. T., Brownell, M. T. y Benedict, A. (2019). Relationships among especial education teachers' knowledge, instructional practice and students' performance in reading fluency. Learning Disabilities Research \& Practice, 34(2), 85-96.

Rico, L. (2013). El método del análisis didáctico. UNIÓN, 33, 11-27.

Rico, L. y Fernández-Cano, A. (2013). Análisis didáctico y metodología de investigación. En L. Rico, J. L. Lupiáñez y M. Molina (Eds.), Análisis 


\section{Universidade Federal da Grande Dourados}

didáctico en educación matemática. (pp. 1-22). Granada, España: Comares.

Rico, L., Lupiáñez, J. L. y Molina, M. (2013). Análisis didáctico en educación matemática. Granada, España: Comares.

UGCI. (2019). Plan de Estudios. Licenciatura en educación y pedagogía en educación especial, especialidad en comunicación y lenguaje, o en educación de personas sordas, o en aprendizaje (Documento de Trabajo UMCE). Santiago, Chile.

Vicerrectoría Académica. (2016). Modelo educativo UMCE. Santiago, Chile: UMCE. 